\title{
Salvage prostatectomy and high risk disease: push your limits - robotics is better
}

\author{
SS Goonewardene*, D Cahill ${ }^{1}$ \\ The Royal Free and UCL, London
}

Received: 15 July, 2016; Accepted: 02 August, 2016; Published: 12 August, 2016

*Corresponding author: Goonewardene SS, the Royal Free Hospital and UCL Partners, London, UK, E-mail: ssg7727@yahoo.co.uk

Keywords: Robotics; Surgery; Salvage therapy; Prostatectomy; High risk

Salvage Robotic Radical Prostatectomy (SRALP) is known for managing more high risk disease. This is a treatment option for biochemical recurrence in prostate cancer [1]. Failure of non-surgical primary treatment for localized disease has rates ranging from $20 \%$ to $60 \%$ [2]. This is an uncommonly performed procedure, due to the technical challenges involved [1]. SRALP is associated with high complication rates for rectal injury, urinary leak anastomotic stricture and incontinence [3]. This leads to distorted anatomy and difficulty with tissue-planes [3]. As a result, more extensive dissection is required. This leads to a higher rate of complications. Given the advances within robotics, this next question should robotic surgery be considered for high risk prostate cancer and what are the technical tips?

Robotic-assisted salvage radical prostatectomy is not widely performed. This has specific advantages over open surgery including 3-D visualisation of tissue planes rather than feel, blood loss and a more secure anastomosis [3].If the degree of dissection is less, outcomes are better. In contrast to open surgery, this requires a great deal of open dissection.

It has also previously been shown, with multivariable analysis, D'Amico risk groups or pathologic Gleason grade, stage, and margins were the strongest predictors of biochemical recurrence in salvage therapy [4]. Detectable PSAs and high risk disease were independent predictors of receipt of salvage therapy [4]. Persistent disease signals the risk of progression likely requiring early salvage treatment [4]. However, if this is detected early, and disease is localised, outcomes will be better.

Part of the explanation for the rapid uptake of robotic surgery is the gentler learning curve compared with laparoscopic radical prostatectomy. Even though robotic, salvage surgery still has potential difficulties, these can be overcome with the robotic platform [5]. Voiding complications while on the steepest part of the learning curve are crucial to overcome [5]. Complex reconstruction of the bladder neck can be achieved with success robotically, in stark contrast to open surgery. Urinary leak is known to be common, but less so than open surgery (39\%) [6]. This is comparable to other robotic series. Although some patients had limited follow-up, 6 (33\%) were continent and 67\% were free of biochemical progression.

Many SRALP patients have localised disease recurrence and therefore potentially curable disease [2]. The key to decreased morbidity SRALP outcomes are to have experienced centers and fellowship training, a standardisation of training [2]. The robotic surgery curriculum is welcomed as part of this. These centre have demonstrated the advantages of the robotic platform in the performance of salvage radical prostatectomy. This includes decreased blood loss, short length of stay and improved haptic feedback. This was confirmed with a decreasing positive surgical margin rate with each consecutive group of 50 cases, including pT3 and high-risk patients [7]. The 3-year, 5-year, and 7-year BCR-free survival rates were $79.2 \%, 75.3 \%$, and $70.2 \%$, respectively [7]. 250 cases have become a landmark for lowest positive surgical margin rates, although, it may take more experience than this to manage SRALP cases [7].

Increasingly complex cases can be taken on as the learning curve progress [5]. Very often with salvage cases, significant intra-abdominal adhesions, dense periprostatic inflammation post URP; large prostate gland size and median lobes may alter bladder neck anatomy. This makes for a difficult urethro- vesical anastomosis [5]. This highlights the importance of a having a good robotic pelvic experience, prior to taking on salvage cases.

Compared to open and laparoscopic procedures perioperative SRALP outcomes were positive, with low complication rates and estimated blood loss equivocal to open or lap procedures [1]. This demonstrates robotic technology can aid the surgeon in salvage prostatectomy [1]. However, experience and training are key to minimal morbidity [3]. This procedure has significant advantages over open surgery in selected patients and is an excellent alternative to other salvage therapies, and allows a broader spectrum of patients to be treated compared to open surgery [3]. SRALP also offers complete staging of high-risk prostate cancer thereby allowing optimum planning of adjuvant and salvage therapies [8]. However, many high-risk prostate cancers are subsequently downgraded or down staged on final histopathology [8], rendering the patient disease free. 
However, SRALP is technically demanding, and experienced surgeons are needed [9]. Post radiation cystitis, fibrosis, and tissue plane obliteration can lead to significant complications, such as rectal injuries, anastomotic stricture, and urinary incontinence [9]. This highlight how functional status of patients before robotassisted salvage prostatectomy maybe compromised [10]. Three of the six patients had extremely poor sexual function before surgery (EPIC sexual domain < 50), and three-quarters had significant irritative symptoms (mean EPIC urinary irritation score 60.5) [10]. There were no intraoperative complications [10]. Of six patients, four (75\%) remain free of disease; however, incontinence and erectile dysfunction were evident in all, to some degree [10]. The outcomes from SRALP have also been confirmed by Pokala et al [11] and also confirmed excellent survival.

SRALP for high risk disease gives good outcomes both ontologically and functionally, however, good fellowship training and experienced surgeons are key.

SS Goonewardene and D Cahill, have no conflicts of interest.

\section{References}

1. Wetherell D, Bolton D, Kavanagh L, Perera M. Current role of salvage robotic-assisted laparoscopic prostatectomy. World Journal of Urology. 2013;31(3):463-469. doi: 10.1007/s00345-013-1025-3.

2. Kaffenberger S, Smith J. Salvage robotic radical prostatectomy. Indian Journal of Urology. 2014;30(4):429-433. doi: 10.4103/09701591.142074.

3. Eldred-Evans D, Sturch P, Cahill D, Dasgupta P, Challacombe B, Popert R. Salvage robotic-assisted radical prostatectomy: Challenges and solutions. Urology. 2013;1:S170.

4. Diaz M, Peabody JO, Kapoor V, Sammon J, Rogers CG, Stricker H, et al. Oncologic outcomes at 10 years following robotic radical prostatectomy. European Urology. 2015;67(6):1168-1176. doi: 10.1016/j.eururo.2014.06.025.

5. Goldstraw MA, Challacombe BJ, Patil K, Amoroso P, Dasgupta P, Kirby RS. Overcoming the challenges of robot-assisted radical prostatectomy. Prostate Cancer and Prostatic Diseases. 2012;15(1):17. doi: 10.1038/pcan.2011.37.

6. Eandi JA, Link BA, Nelson RA, Josephson DY, Lau C, Kawachi MH, et al. Robotic Assisted Laparoscopic Salvage Prostatectomy for Radiation Resistant Prostate Cancer. Journal of Urology. 2010;183(1):133-137. doi: 10.1016/j.juro.2009.08.134.

7. Ou YC, Yang CK, Chang KS, Wang J, Hung SW, Tung MC, et al. The surgical learning curve for robotic-assisted laparoscopic radical prostatectomy: Experience of a single surgeon with 500 cases in Taiwan, China. Asian Journal of Andrology. 2014;16(5):728-734. doi: 10.4103/1008-682X.128515.

8. Murphy D. State of the art lecture 9-the contemporary role of surgery in the management of high-risk prostate cancer. International Journal of Urology. 2012;19:229.

9. Rocco B, Cozzi G, Spinelli MG, Grasso A, Varisco D, Coelho RF, et al. Current status of salvage robot-assisted laparoscopic prostatectomy for radiorecurrent prostate cancer. Current Urology Reports. 2012;13(3):195-201. doi: 10.1007/s11934-012-0245-1.

10. Strope SA, Coelho M, Wood DP, Hollenbeck BK. Robot-assisted salvage prostatectomy: evaluation of initial patient-reported outcomes. Journal of Endourology. 2010;24(3):425-427. doi: 10.1089/ end.2009.0143.

11. Pokala N, Huynh DL, Henderson AA, Johans C. Survival Outcomes in Men Undergoing Radical Prostatectomy After Primary Radiation Treatment for Adeno carcinoma of the Prostate. Clin Genitourin Cancer. 2016;14(3):218-225. doi: 10.1016/j.clgc.2015.12.010. 